\title{
Propiedades organolépticas, físicas y mecánicas de la madera de Cordia alliodora (R. y P.) Oken de parcelas agroforestales en Jaén, Cajamarca
}

\section{Organoleptic, physical and mechanical properties of the wood of Cordia alliodora (R. and P.) Oken of agroforestry plots in Jaén, Cajamarca}

Franklin H Fernandez $\mathrm{Z}^{1}$., José A León $\mathrm{M}^{2}$., Annick E Huaccha $\mathrm{C}^{3}$. y Francisco F Aguirre $\mathrm{R}^{4}$.

\section{RESUMEN}

Cordia alliodora (R. y P.) Oken, cumple con las características para convertirse en una de las especies maderables más importantes usada en sistemas agroforestales en la zona nor-oriental del Perú. En esta área se encuentra madera de calidad, con alto valor económico y rápido crecimiento; la escasez de estudios referidos a las propiedades tecnológicas de esta madera han traído como consecuencia que no se realice un aprovechamiento y transformación, a la misma escala a la que se está produciendo; por ello establecimos las propiedades organolépticas, físicas y mecánicas de la madera de $C$. alliodora, de plantaciones agroforestales; se realizó el corte de muestras de 15 árboles, de diferentes edades. plantaciones agroforestales Las Naranjas, Jaén Los ensayos siguieron la norma técnica peruana (NTP): NTP 251.010, NTP 251.011, NTP 251.012, NTP 251.014, NTP 251.015, NTP 251.016, NTP 251.017. Los resultados obtenidos muestran que únicamente para la contracción total longitudinal existen diferencias estadísticamente significativas entre los distintos tratamientos evaluados (edad de los árboles). Así mismo, por la densidad básica, contracciones totales y resistencia mecánica puede incluirse en el grupo de especies de baja densidad.

Palabras clave: Cordia alliodora, propiedades físico-mecánicas, características organolépticas, plantaciones agroforestales.

\begin{abstract}
Cordia alliodora (R. and P.) Oken, meets the characteristics to become one of the most important timber species used in agroforestry systems in the north-eastern of Peru. In this area has quality wood, with high economic value and rapid growth; the scarcity of studies referring to the technological properties of this wood have resulted in a lack of exploitation and transformation, at the same scale at which it is being produced; for this reason we have established the organoleptic, physical and mechanical properties of the wood C. alliodora, of agroforestry plantations; The samples were cut from 15 trees, of different ages. Agroforestry plantations from Las Naranjas, Jaén the trials followed the Peruvian technical standard (NTP): NTP 251.010, NTP 251.011, NTP 251.012, NTP 251.014, NTP 251.015, NTP 251.016, NTP 251.017. The results obtained show that only for total longitudinal contraction there are statistically significant differences between the different treatments evaluated (age of the trees). Likewise, due to the basic density, total contractions and mechanical resistance it can be included in the group of low-density species.
\end{abstract}

Keywords: Cordia alliodora (R. and P.) Oken, Physical-mechanical properties, organoleptic characteristics, agroforestry plantations.

\footnotetext{
${ }^{1}$ Universidad Nacional de Jaén, Perú. Email: frank9615@gmail.com

${ }^{2}$ Universidad Nacional de Jaén, Perú. Email: jandreslm1@gmail.com

${ }^{3}$ Universidad Nacional de Jaén, Perú. Email: annick_huaccha@unj.edu.pe

${ }^{4}$ Cooperativa de Servicios Múltiples Sol\&Café, Perú. Email: faguirredelosrios@ gmail.com
} 


\section{INTRODUCCIÓN}

El conocimiento de las propiedades físico-mecánicas de la madera juega un rol importante en el momento de determinar su uso tanto en la industria de la construcción como en la de muebles (Yosa, Baradit y Acevedo, 2015).

C. alliodora, además de sus propiedades maderables, es apreciada por su abundante repoblación natural, proveedora de sombra para los cultivos, autopoda eficiente, promotora del reciclaje de nutrimentos, constituir un ingreso económico adicional cuando baja la producción o los precios del café y tener un rápido crecimiento (Glover y Beer, 1986; Albertin y Nair 2004).

Las maderas de plantaciones de Jaén son heterogéneas como en América Central. (Aguirre y Zevallos, 2014), el color determina su uso, diferenciándose dos tipos, blanco y negro (Dalwitz, 2000). El Laurel blanco y Laurel Negro son maderables de importancia en Jaén, comercializada muy bien en ese mercado (Aguirre y Zevallos, 2014).

Aguirre y Zevallos (2014) determinaron que la densidad del laurel blanco $0,31 \mathrm{~g} / \mathrm{cm}^{3}$, clasificada como madera liviana, mientras que Barrance, et al (2003) estableció que la densidad básica varia de 0.34 a $0.64 \mathrm{~g} / \mathrm{cm}^{3}$ esto variara entre árboles y sitio, ya que en zonas secas la madera es más pesada. Tenorio et al. (2016) determinaron que la contracción total tangencial y la contracción total radial para Cordia alliodora de 14 años fue de $5.6 \%$ y $3.9 \%$ respectivamente.

Las provincias de Jaén y San Ignacio, en el norte de Perú albergan 26579 ha. y 17748.5 ha de café respectivamente, y aproximadamente el 80 y $82 \%$ del total se encuentran sembradas bajo sombra (Ministerio de Agricultura, 2003). En la zona uno de los árboles que más destaca en los sistemas agroforestales es C. alliodora, conocida localmente como "Laurel"; actualmente, estos árboles se encuentran disponibles para su aprovechamiento, convirtiéndose en una de las maderas de especies nativas más importantes en esta región.

A pesar de la abundancia e importancia de esta especie maderable, no se tiene conocimiento de la calidad de la madera producida, por la ausencia de estudios relacionados a la tecnología de la madera; limitando su aprovechamiento y transformación a la misma escala que se está produciendo, y esto se refleja en los datos reportados por el Ministerio de Agricultura (2010) donde reporta que en el departamento de Cajamarca en el año 2009 solo se aprovechó $125.39 \mathrm{~m}^{3}$ de madera rolliza de Laurel. Generar información sobre aspectos tecnológicos de la madera producida en estos sistemas agroforestales, ayudará al planificar adecuadamente su transformación y comercialización de acuerdo con sus propiedades físicas, organolépticas y mecánicas. En ese contexto, el objetivo de este estudio fue describir las propiedades organolépticas, físicas y mecánicas de la madera de $C$. alliodora proveniente de plantaciones agroforestales. 


\section{MATERIAL Y MÉTODOS}

\section{Área de estudio}

La zona de estudio está localizada en la región Cajamarca, provincia y distrito de Jaén en el Centro Poblado San Miguel de las Naranjas, de acuerdo a la división de las ocho regiones naturales del Perú, presentada por la tesis del Geógrafo (Pulgar, 1941), esta zona de encuentra en la región Yunga siendo tierras de clima cálido de los valles y quebradas que suben los andes, mientras que según la clasificación de Koppen (1948) estos climas son de carácter templado con lluvias templadas y clima tropical permanentemente húmedo, la temperatura en las zonas más bajas oscila $\operatorname{los} 24^{\circ} \mathrm{C}$ y en las zonas más elevadas en invierno va desde los 10 a $18{ }^{\circ} \mathrm{C}$ con una precipitación anual mayor a 750 $\mathrm{mm}$.

\section{Selección de árboles muestreados}

El material experimental provino de árboles seleccionados al azar, a razón de cinco árboles por cada grupo de edad (05 a 10 años, 11 a 15 años y 16 a 20 años), sobre los cuales se desarrolló un muestreo destructivo sistematizado para tomar material necesario para el desarrollo de las pruebas físico-mecánicas.

\section{Obtención de trozas}

Se talaron los árboles seleccionados, luego se extrajo una troza de $2 \mathrm{~m}$ de longitud, de ésta, se cortó una vigueta de madera, de $8 \mathrm{~cm}$ de espesor en la sección transversal, las cuales se enumeraron por el número del árbol y la troza correspondiente, los cortes fueron sellados con pintura metálica para mantener el contenido de humedad, posteriormente se realizaron las orientaciones y cortes adecuados de la vigueta para la extracción de las probetas que fueron sometidas a los ensayos físico - mecánicos.

\section{Variables evaluadas}

\section{Propiedades físicas}

Las propiedades físicas analizadas fueron: contenido de humedad, densidad y contracción total. Estas propiedades se analizaron siguiendo las siguientes Normas Técnicas Peruanas (NTP), NTP 251.010: Método de determinación del contenido de humedad, NTP 251.011: Método de determinación de la densidad, NTP 251.012: Método de determinación de la contracción.

\section{Propiedades mecánicas}

Las propiedades mecánicas analizadas fueron: cizallamiento paralelo al grano, compresión perpendicular y paralela al grano, dureza y flexión estática; todos los ensayos se hicieron siguiendo 
los lineamientos de las Nomas Técnicas Peruanas (NTP). NTP 251.013: Método de determinación del cizallamiento paralelo al grano, NTP 251.014: Método de determinación de la compresión paralela al grano, NTP 251.015: Método de determinación de la dureza, NTP 251.016: Método de determinación de la compresión perpendicular, NTP 251.017: Método de determinación de la flexión estática.

\section{Procesamiento de datos y análisis de resultados}

Se hizo un análisis básico de los datos, elaborando tablas y gráficos, además de la obtención de promedios y coeficientes de variación. El análisis, se realizó sobre tres tratamientos (5-10 años, 1115 años y 16-20 años). Se realizó un Diseño completamente al azar con sub muestreo (DCA), se realizaron los cálculos de ANVA, solo para los tratamientos que tuvieron estadísticas significativas $(\mathrm{P}<0.05)$ se utilizó la prueba de Duncan.

\section{RESULTADOS Y DISCUSIÓN}

\section{Propiedades organolépticas}

Se pudo apreciar el color de la albura amarillo y crema, color del duramen oscuro, transición de color entre albura y duramen de forma abrupta, veteado en forma de arcos superpuestos, olor aromático, grano entrecruzado y textura fina. Pérez (2014), afirma que en ocasiones, la zona de transición entre la albura y el duramen no está bien definida, el veteado es pronunciado debido a la diferencia de tono entre madera temprana y madera tardía de cada anillo de crecimiento y también a las líneas de vaso. La albura es de color pardo muy pálido (HUE 10 YR 8 \% 3), amarillento o café pálido. Es una madera con textura uniforme de fina a mediana, tiene mucho lustre, el hilo es generalmente recto, aunque a veces estrellado.

\section{Propiedades físicas y mecánicas}

No existen diferencias estadísticamente significativas para los tres tratamientos evaluados $(5-10$ años, 11 - 15 años y de 16 - 20 años), excepto para la contracción total longitudinal (Tabla 3), además se observan que los coeficientes de variabilidad para las distintas contracciones totales de la madera de Cordia alliodora (R. y P.) Oken se encuentran bajo los límites aceptables. 
Tabla 1. Análisis de varianza para la contracción total de la madera de Cordia alliodora (R. y P.) Oken, a un intervalo de confianza del $95 \%$.

\begin{tabular}{|c|c|c|c|c|c|c|}
\hline \multirow{2}{*}{$\begin{array}{l}\text { Fuentes de } \\
\text { variación }\end{array}$} & \multirow{2}{*}{$\begin{array}{l}\text { Grados de } \\
\text { libertad }\end{array}$} & \multicolumn{4}{|c|}{ Contracción Total } & \multirow{2}{*}{$\begin{array}{c}\mathbf{F} \\
\text { tabula } \\
\mathbf{r}\end{array}$} \\
\hline & & $\begin{array}{c}\text { C. } \mathbf{T} \text {. } \\
\text { Tangencial } \\
\text { F }\end{array}$ & $\begin{array}{c}\text { C. T. Radial } \\
\text { F }\end{array}$ & $\begin{array}{c}\text { C. T. } \\
\text { Longitudinal } \\
\text { F }\end{array}$ & $\begin{array}{c}\text { C. T. } \\
\text { Volumétr } \\
\text { ica } \\
\text { F }\end{array}$ & \\
\hline Tratamientos & 2 & 0.8597 N. S & 2.2594 N. S & $4.2023^{*}$ & $\begin{array}{c}1.1809 \mathrm{~N} . \\
\mathrm{S}\end{array}$ & 3.885 \\
\hline $\begin{array}{l}\text { Error experimental } \\
\text { Error de muestreo } \\
\text { Total }\end{array}$ & $\begin{array}{l}12 \\
45 \\
59\end{array}$ & & & & & \\
\hline $\begin{array}{ll}\text { Coeficiente } & \text { de } \\
\text { variabilidad (\%) }\end{array}$ & & 7.4313 & 11.3818 & 10.3475 & 7.4058 & \\
\hline
\end{tabular}

N. S: No significativo. *: Significativo. C. T.: Contracción total

Tabla 2. Prueba de Duncan para los datos de contracción total longitudinal de la madera de Cordia alliodora (R. y P.) Oken.

\begin{tabular}{lcc}
\hline \multicolumn{1}{c}{ Tratamiento } & Media & Significancia \\
\hline $5-10$ años & 0.76 & $\mathrm{~A}$ \\
$16-20$ años & 0.60 & $\mathrm{Ab}$ \\
$11-15$ años & 0.52 & $\mathrm{~B}$ \\
\hline
\end{tabular}

Los resultados estadísticos de la prueba de Duncan nos indican la existencia de diferencias significativas entre el primer y el segundo tratamiento, este comportamiento de la madera se les atribuye a características intrínsecas de las probetas en estudio como por ejemplo la presencia de madera juvenil, ya que esta tiene mayor ángulo de depósito de las microfibrillas en la capa S2 de la pared secundaria, tal como lo señala Vásquez y Ramírez (2005).

Tabla 3. Resumen de los ensayos realizados por grupos de edad de la madera de Cordia alliodora (R. y P.)

Oken

\begin{tabular}{|c|c|c|c|}
\hline \multirow[t]{2}{*}{ Ensayo } & \multicolumn{3}{|c|}{ Edad de los árboles } \\
\hline & 5-10 años & 11-15 años & 16-20 años \\
\hline \multicolumn{4}{|l|}{ Dureza } \\
\hline Dureza (lateral) $\left(\mathrm{Kg} / \mathrm{cm}^{2}\right)$ & 264.81 & 266.87 & 273.14 \\
\hline Dureza (axial) $\left(\mathrm{Kg} / \mathrm{cm}^{2}\right)$ & 251.84 & 260.38 & 261.54 \\
\hline
\end{tabular}

Contracción normal 


\begin{tabular}{|c|c|c|c|}
\hline C. Tangencial (\%) & 3.48 & 3.77 & 3.90 \\
\hline C. Radial (\%) & 2.52 & 2.52 & 2.30 \\
\hline C. Longitudinal (\%) & 0.32 & 0.26 & 0.32 \\
\hline C. Volumétrica (\%) & 4.93 & 4.38 & 4.50 \\
\hline \multicolumn{4}{|l|}{ Contracción total (\%) } \\
\hline C. Tangencial (\%) & 5.30 & 5.75 & 5.99 \\
\hline C. Radial (\%) & 3.65 & 3.73 & 3.07 \\
\hline C. Longitudinal (\%) & 0.76 & 0.52 & 0.60 \\
\hline C. Volumétrica (\%) & 9.15 & 8.49 & 8.26 \\
\hline Cizallamiento tangencial $\left(\mathrm{Kg} / \mathrm{cm}^{2}\right)$ & 52.77 & 56.37 & 60.61 \\
\hline \multicolumn{4}{|l|}{ Compresión paralela } \\
\hline $\operatorname{ELP}\left(\mathrm{Kg} / \mathrm{cm}^{2}\right)$ & 131.61 & 135.23 & 154.69 \\
\hline $\operatorname{MOR}\left(\mathrm{Kg} / \mathrm{cm}^{2}\right)$ & 175.35 & 191.45 & 181.79 \\
\hline $\operatorname{MOE}\left(\mathrm{Kg} / \mathrm{cm}^{2}\right)$ & 59445.90 & 73868.20 & 69667.10 \\
\hline \multicolumn{4}{|l|}{ Compresión perpendicular } \\
\hline $\operatorname{ELP}\left(\mathrm{Kg} / \mathrm{cm}^{2}\right)$ & 21.46 & 22.10 & 24.07 \\
\hline \multicolumn{4}{|l|}{ Flexión estática } \\
\hline $\operatorname{ELP}\left(\mathrm{Kg} / \mathrm{cm}^{2}\right)$ & 228.05 & 236.06 & 256.61 \\
\hline $\operatorname{MOR}\left(\mathrm{Kg} / \mathrm{cm}^{2}\right)$ & 449.50 & 470.98 & 474.66 \\
\hline $\operatorname{MOE}\left(\mathrm{Kg} / \mathrm{cm}^{2}\right)$ & 81479.48 & 93829.48 & 104603.20 \\
\hline \multicolumn{4}{|l|}{ Densidad } \\
\hline Saturada $\left(\mathrm{gr} / \mathrm{cm}^{3}\right)$ & 0.76 & 0.76 & 0.71 \\
\hline Seca $\left(\mathrm{gr} / \mathrm{cm}^{3}\right)$ & 0.40 & 0.41 & 0.42 \\
\hline Anhidra $\left(\mathrm{gr} / \mathrm{cm}^{3}\right)$ & 0.38 & 0.39 & 0.40 \\
\hline Básica $\left(\mathrm{gr} / \mathrm{cm}^{3}\right)$ & 0.36 & 0.35 & 0.36 \\
\hline
\end{tabular}

Los datos de dureza lateral de la madera de Cordia alliodora (R. y P.) Oken, nos permiten ubicarla en el grupo II, dentro de las maderas de clasificación de baja resistencia a la dureza, según lo propuesto por Aróstegui (1982). Nótese que los valores de dureza muestran la tendencia de incrementarse conforme lo hace la edad, obteniéndose los valores más altos en el tercer tratamiento, lo que se explica por su mayor densidad, puesto que existe una alta correlación entre la dureza y densidad de la madera (Forest Products Laboratory, 2010; Rodríguez et al., 2014).

Se puede observar que únicamente para la contracción total tangencial muestra una tendencia a incrementarse conforme la edad va aumentando, la contracción total tangencial también lo hace, sin embargo esto no se observa en los demás tipos de contracción total, estos últimos resultados podrían atribuirse a la presencia de madera juvenil con posibles zonas de madera de reacción esto estaría 
provocando una distribución y severidad anormal de la contracción entre las diferentes muestras (Pantigoso, 2009), además podría explicarse por la mayor inclinación de las microfibrillas en la madera juvenil (Torres, 2009).

Tenorio et al. (2016) determinaron que la contracción total tangencial y la contracción total radial para Cordia alliodora de 14 años fue de $5.6 \%$ y $3.9 \%$ respectivamente, datos similares a los registrados en la presente investigación, esto se debe a las similitudes edafoclimáticas de las zonas de ambos estudios donde crece la especie Cordia alliodora, según los resultados de contracción volumétrica la cual está entre $8.26 \%$ y $9.15 \%$, pertenece al grupo II, dentro de las maderas de baja contracción volumétrica, según lo propuesto por Aróstegui (1982).

El cizallamiento paralelo al grano de la madera de Cordia alliodora (R. y P.) Oken está relacionada con la edad de esta especie, al igual que con la densidad; es decir, mientras mayor es la densidad, la resistencia al cizallamiento se incrementa, tal como lo afirma Condori (2007).

En el cizallamiento tangencial de la madera de Cordia alliodora (R. y P.) Oken, los dos primeros tratamientos la ubican en el grupo II, dentro de las maderas de clasificación de baja resistencia, mientras que el tercer tratamiento está en límite inferior de las maderas ubicadas en el III grupo considerada como maderas de media resistencia, según lo propuesto por Aróstegui (1982), esto se atribuye a la presencia de madera de tensión en las probetas ensayadas, tal como lo señala Sueros (2017).

En comprensión paralela, los datos del esfuerzo al límite proporcional (ELP), muestran tendencia de incrementar con respecto al incremento de la edad de los árboles, mientras que los resultados de módulo de elasticidad (MOE) y del módulo de ruptura (MOR) no muestran esa tendencia, este comportamiento podría ser el resultado de una combinación de factores anatómicos con la presencia de madera juvenil (Silva, 2007), durante la selección de listones para la preparación de probetas, además de factores edafoclimáticos que hacen que el desarrollo de los árboles no sean iguales, afectando la formación, ubicación, tamaño de los espacios porosos; tal como lo señala Drew (1998) que las especies vegetales tienen una periodicidad de producción de células, regulada por un patrón anual de disponibilidad de agua, lo que sugiere la formación anual de cada anillo en la madera.

En comprensión perpendicular al grano de la madera de C. alliodora, el esfuerzo al límite proporcional (ELP) y módulo de elasticidad (MOE), siguen la tendencia de incrementar con respecto al incremento de la edad, esto se asocia a la densidad de la madera, es decir, mientras mayor es la densidad, el valor de estas propiedades también incrementa (Urbina 2017). Condori 
(2007), afirma que toda propiedad resistente de un material depende en gran medida de la cantidad de materia sólida que posea por unidad de volumen; es decir, la densidad de éste, así; las propiedades de las probetas libres de defectos deben de tener una buena correlación con la densidad básica y por ende con la edad de los árboles, tal como lo señalan Hocker (1984) quien afirma que la densidad de la madera depende principalmente de la edad y Daniel et al. (1982) quien explica que a mayor edad se forman anillos con mayor porcentaje de madera tardía y ello incrementa la densidad de la madera en la parte más externa del árbol y por ende incrementa el valor promedio en los árboles más adultos, de la misma forma Bhat et al. (2001) indica que el valor de la densidad básica tiende a aumentar con la edad del árbol, al mismo tiempo que deben considerarse factores como la formación de madera juvenil, que en el caso de la madera joven de $C$. alliodora es un factor que influencia directamente su comportamiento.

En flexión estática de la madera de Cordia alliodora (R. y P.) Oken, el módulo de ruptura (MOR) va de 470.98 a $449.50 \mathrm{Kg} / \mathrm{cm}^{2}$, lo que la ubica en el grupo II, dentro de las maderas de clasificación de baja resistencia según lo propuesto por Aróstegui (1982), esta condición podría asociarse como menciona Niva (2006) y, Pearson y Gilmore citado por Cown (1992) a la presencia de la madera juvenil con mayor ángulo microfibrilar atribuido por ser una especie de rápido crecimiento.

La densidad básica de la madera de C. alliodora, se encuentra entre 0.35 y $0.36 \mathrm{~g} / \mathrm{cm}^{3}$, considerada entre maderas de baja densidad según lo propuesto por Aróstegui (1982), por su parte, Tenorio et al. (2016) determinaron que la densidad básica para Cordia alliodora de 14 años fue de $0.34 \mathrm{~g} / \mathrm{cm}^{3}$, Aguirre y Zevallos (2014) determinaron que la densidad del laurel blanco $0,31 \mathrm{~g} / \mathrm{cm}^{3}$, clasificada como madera liviana, mientras que Barrance, et al (2003) estableció que la densidad básica varia de 0.34 a $0.64 \mathrm{~g} / \mathrm{cm}^{3}$, estas variaciones de densidad entre los distintos estudios se debe a características intrínsecas de los árboles sometidos al ensayo, dentro de las cuales podemos destacar la precipitación, ya que en zonas más secas la madera es más pesada con respecto a zonas húmedas.

Para la densidad en estado saturado de la madera de Cordia alliodora (R. y P.) Oken, Tenorio et al. (2016) determinaron que esta densidad para Cordia alliodora de 14 años fue de $0.85 \mathrm{~g} / \mathrm{cm}^{3}$, dato superior al encontrado en la presente investigación, esto podría atribuirse a características inherentes de las probetas estudiadas (Vilcayahuri, 2009). Los datos de densidad en estado seco al aire y estado anhidro de la madera de Cordia alliodora (R. y P.), muestran una tendencia de incrementarse conforme se incrementa la edad de los árboles, tal como lo señala Quintana et al. (2011), al respecto, Bisset y Dadswell (1950) concluyen que al inicio de un periodo vegetativo 
existe una intensa actividad de divisiones cambiales, lo que produce células más cortas, pero al disminuir esta actividad cambial las células formadas tienen más tiempo para desarrollarse en tamaño Según Aloni (1991), la presencia de vasos con diámetro amplio se debe a una rápida diferenciación celular en los primeros momentos de reactivación del cambium vascular, mientras que la presencia de vasos más estrechos ocurre cuando la diferenciación es más lenta, originando que la pared secundaria se forme primero e impida un ensanchamiento posterior de la célula.

\section{CONCLUSIONES}

A partir de la densidad básica, contracciones totales y resistencia mecánica (MOR), se puede ubicar a la madera de $C$. alliodora (R. y P.) Oken proveniente de plantaciones agroforestales en el grupo II dentro de las maderas de baja densidad y resistencia.

Según las propiedades organolépticas, físicas y mecánicas de la madera de $C$. evaluada, se recomienda que se use en obras de interiores (tabiquería, cielo raso), chapas (decorativas y compensadas), cajonería, mobiliario general (bancas, mesas, reposteros, armarios, carpetas, muebles pintados). Los resultados de dureza, contracción normal tangencial y radial, contracción total tangencial, cizallamiento tangencial, comprensión perpendicular, flexión estática y densidad nos muestran una relación directa con la edad de los árboles evaluados.

Los resultados aplican, principalmente, para la zona del Centro Poblado San Miguel de las Naranjas, Jaén y las características agroforestales de la zona, debido a que las propiedades tecnológicas de la madera pueden ser afectada por las condiciones edafoclimáticas.

\section{AGRADECIMIENTOS}

A la Cooperativa Sol\&Café LTDA por el apoyo económico brindado y a la Universidad Nacional Agraria La Molina con las orientaciones del Ing. Gerardo Alarcón Cubas.

\section{REFERENCIAS BIBLIOGRÁFICAS}

Aguirre, F. (2008). Caracterización Fenotípica, dendrológica y anatómica de los tipos morfológicos de árboles de Cordia alliodora (R. y P.) Oken procedentes de plantaciones realizadas en Jaén, Cajamarca - Perú. Tesis para optar el grado de: Magister Scientiae. Universidad nacional Agraria la Molina. Lima, Perú. 54 pp. 
Albertin, A. y Nair, P. (2004). Farmers' perspectives on the role of shade trees in coffee production systems: An assessment from the Nicoya Peninsula, Costa Rica. Human ecology, 32(4):443463.

Aloni, R. (1991). Wood formation in deciduous hardwood trees. In: Offprints from Physiology of Trees. Raghavandra, A.S. (ed.). John Wiley \& Sons, Inc. New York, USA. pp: 175-196

Aróstegui, A; Díaz, M. (1992). Propagación de especies forestales nativas promisorias en Jenaro Herrera. Iquitos, PE, IICA 119 p.

Aróstegui, A. (1982). Recopilación y análisis de estudios tecnológicos de maderas peruanas. Lima, PE, Proyecto PNUD/FAO/PER/71/511. 57 p. (Documento de Trabajo W2)

Aróstegui, A (1974). Estudio tecnológico de maderas del Perú (Zona Pucallpa): Características tecnológicas y usos de la madera de 145 especies del país volumen 1. Lima, PE, UNALM. 483 p.

Barrance, A., Beer, J., Boshier, D., Chamberlain, J., Cordero, J., Detlefsen, G., . . Pennington, T. (2003). Arboles de Centro America. Costa Rica: Turrialba.

Bhat, K. (1998). Properties of fast-grown teakwood: impact on end-users requirements. Journal of Tropical Forest Science 4(1):1-10.

Bisset, I., and Dadswell. H. (1950) The variation in cell length within one growth ring of certain angyosperms and gymnosperms. Austr. Forestry 14: 17-29.

Boshier, D.H. (2002). Cordia alliodora (Ruiz y Pav.) Oken, in Tropical Trees Seed Manual, USDA Forest Service, Agriculture Handbook 721. 411-413.

CATIE. (1994). Laurel (Cordia alliodora R y P) Oken, especie de árbol de uso múltiple en América central. Turrialba, Costa Rica, CATIE. 47 p. (Serie Técnica. Informe Técnico nº. 239).

CONAFOR, (2007). Fichas técnicas sobre características tecnológicas y usos de maderas comercializadas en México. México. 132 p.

Condori, C. (2007). Propiedades mecánicas de Cedrelinga cateniformis Ducke (Tornillo) proveniente de dos tipos de plantaciones y diferentes edades del Centro de Investigación Jenaro Herrera - Loreto. Tesis para optar el título de ingeniero forestal. Universidad Nacional Agraria La Molina. 124 p.

Daniel T., Helms, J. y Baker. F. (1982). Principios de silvicultura. Mc Graw-Hill. México. 492 p.

Drew, A. (1998). Growth rings, phenology, hurricane disturbance and climate in Cyrilla racemifolia L., a rain forest tree of the Luquillo Mountains, Puerto Rico. Biotropica 30: 35-49. 
Glover, N. y Beer, J. (1986). Nutrient cycling in two traditional Central American agroforestry systems. Agroforestry Systems, 4(2):77-87.

Hocker, J. (1984). Introducción a la biología forestal. A.G.T. Editor, S.A. México. 431 p.

León, H. (2003). Anatomía De La Madera De 9 Especies Del Género Cordia L. (Boraginaceae Cordioideae) Que Crecen En Venezuela. Revista Forest. Venez.. 47(2): 83-94.

López, J. (2006). Propiedades físico-mecánicas del Aliso, Alnus acuminata HBK, proveniente de Chalaco-Piura. Tesis para optar el título de ingeniero forestal. Universidad Nacional Agraria La Molina. 106 p.

Ministerio de Agricultura. (2003). Programa para el desarrollo de la Amazonía. Caracterización de las zonas cafetaleras en el Perú. Lima.136 p. Disponible en: http://www.infocafes.com/descargas/biblioteca/94.pdf

Ministerio de Agricultura. (2010). Perú forestal en números año 2009. Lima. 86 pp. Disponible en: file://C:/Users/User/Downloads/2070.pdf

Pantigoso, J. (2009). Propiedades físicas y mecánicas de la capirona (Calycophyllum spruceanum (Benth) hook ex Schumann) procedente de una plantación experimental en San Alejandro Ucayali-Perú. Tesis para optar el título de ingeniero forestal. Universidad Nacional Agraria La Molina. 140 p.

Pérez, C. (1954). Estudio forestal del laurel, Cordia alliodora (R. \& P.)" Cham., en Costa Rica. Tesis, Instituto Interamericano de Ciencias Agrícolas. Turrialba, Costa Rica, 209 p.

Quintana, S.; Cabudivo, A.; Espíritu, J. y Cabudivo, J. (2011). Propiedades físico-mecánicas de las maderas de Simarouba amara (Aubl.) y Cedrelinga cateniformis (Ducke) de plantaciones de diferentes edades, San Juan Bautista, Loreto, Perú. Con. Amaz.

Rodríguez, R., Zamora, Juan., Silva, J., Salcedo, E. y Fuentes, F. (2014). Propierteies of the Wood fron comercial teak plantations. Universidad de Guadalajara, $12-25$.

Silva, J. (2007). Fichas técnicas sobre características tecnológicas y usos de maderas comercializadas en México. Jalisco, Mexico. Comisión Nacional Forestal (Conafor). p. 45-46

Somarriba, E., Trivelato, M., Villalobos, M., Suárez, A., Benavides, P., Morán, K., ... López, A. (2003). Diagnóstico agroforestal de pequeñas finca cacaoteras orgánicas de Indígenas Bribri y Cábecar de Talamanca, Costa Rica. Agroforestería en las Américas. 10(37-38):24-30.

Suárez, A. y Somarriba, C. (2002). Aprovechamiento sostenible de madera de Cordia alliodora de regeneración natural en cacaotales y bananales de indígenas de Talamanca, Costa Rica. Agroforestería en las Américas, 9(35):50-54. 
Sueros, C. (2017). Análisis de las propiedades mecánicas de la madera de raleo de Guazuma crinita (Bolaina Blanca) de tres edades. Tesis para optar el título profesional de ingeniero forestal. Universidad Nacional Agraria La Molina. Lima, Perú. 96 p.

Tenorio, C., Moya, R., Salas, C., y Berrocal, A. (2016). Evaluation of wood properties from six native species of forest plantations in Costa Rica. Bosque, 37(1), 71-84.

Torres, F. (2009). Evaluación de las propiedades físico-mecánicas de la especie Pino chuncho (Schizolobium amazonicum Huber ex Ducke) proveniente de plantaciones del Bosque Nacional Alexander Von Humboldt-Ucayali. Tesis para optar el título de ingeniero forestal. Universidad Nacional Agraria La Molina. 141 p.

Urbina, L. (2017). Propiedades mecánicas de la madera de raleo procedente de plantaciones de cuatro años de Guazuma crinita Martius (Bolaina blanca) - Huánuco. Tesis para optar el título de ingeniero forestal. Universidad Nacional Agraria La Molina. 84 p.

Vásquez, A. y Ramírez, A. (2005). Maderas comerciales en el Valle de Aburrá: Área Metropolitana del Valle de Aburrá. Medellín, Colombia. p 246. 